\title{
Hepatic bile acids and bile acid-related gene expression in pregnant and lactating rat
}

Background /Aim: Significant physiological changes occur during pregnancy and lactation. Intrahepatic cholestasis of pregnancy (ICP) is a liver disease closely related to disruption of bile acid homeostasis. The objective of this study was to examine the regulation of bile acid metabolism and transport in normal pregnant and lactating rats. Materials and Methods: Livers from timed pregnant SD rats were collected on gestational days (GD) 10, 14 and 19, and postnatal days (PND) 1,7,14 and 21. Total bile acids were determined by the enzymatic method, total RNA was isolated and subjected to real time RT-PCR analysis. Liver protein was extracted for western-blot analysis. Results: Under physiological conditions hepatic bile acids were not elevated during pregnancy but increased during lactation in rats. Bile acid synthesis rate-limiting enzyme Cyp7a1 was unchanged in gestations days, but increased on PND14 and21 at mRNA and protein levels. Expression of Cyp8b1, Cyp27a1 and Cyp7b1 was also higher during lactation. The mRNA levels of small heterodimer partner (SHP) and protein levels of farnesoid X receptor (FXR) were increased during pregnancy and lactation. Bile acid transporters Ntcp, Bsep, Mrp3 and Mrp4 were lower at gestation, but increased during lactation. Hepatic Oatp transporters were decreased during pregnancy and lactation. Conclusion: Hepatic bile acid homeostasis is maintained during normal pregnancy in rats , probably through the FXR-SHP regulation. The expression of bile acid synthesis genes and liver bile acid accumulation were increased during lactation, together with increased bile acid efflux transporter Bsep, Mrp3 and Mrp4 . 


\section{Hepatic bile acids and bile acid-related gene expression in pregnant and}

\section{2 lactating rats}

3 Qiong $\mathrm{N} \mathrm{Zhu}^{1}$, Hong $\mathrm{M} \mathrm{Xie}^{2}$, Dan Zhang ${ }^{1}$, Jie $\mathrm{Liu}^{1,3^{*}}$ and Yuan $\mathrm{F} \mathrm{Lu}^{1 *}$

$4 \quad{ }^{1}$ Department of Pharmacology and Key Lab of Basic Pharmacology of Guizhou, Zunyi Medical

5 College, Zunyi 563003, China.

$6 \quad{ }^{2}$ Department of Gynaecology and Obstetrics, The Third Affiliated Hospital of Zunyi Medical

7 College; Zunyi 563003, China.

$8 \quad{ }^{3}$ University of Kansas Medical Center, Kansas City, KS 66160.

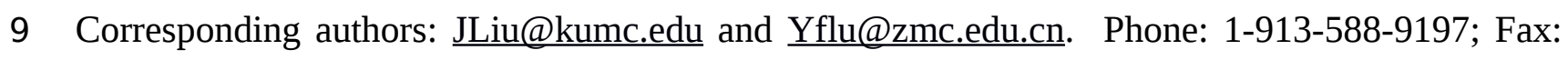

10 011-86-852-860-9033. 


\section{Abstract}

12 Background /Aim: Significant physiological changes occur during pregnancy and lactation.

13 Intrahepatic cholestasis of pregnancy (ICP) is a liver disease closely related to disruption of bile

14 acid homeostasis. The objective of this study was to examine the regulation of bile acid synthesis

15 and transport in normal pregnant and lactating rats. Materials and Methods: Livers from timed

16 pregnant SD rats were collected on gestational days (GD) 10, 14 and 19, and postnatal days

17 (PND) 1,7,14 and 21. Total bile acids were determined by the enzymatic method, total RNA was

18 isolated and subjected to real time RT-PCR analysis. Liver protein was extracted for western-blot

19 analysis. Results: Under physiological conditions hepatic bile acids were not elevated during

20 pregnancy but increased during lactation in rats. Bile acid synthesis rate-limiting enzyme Cyp7a1

21 was unchanged in gestations days, but increased on PND14 and21 at mRNA and protein levels.

22 Expression of Cyp8b1, Cyp27a1 and Cyp7b1 was also higher during lactation. The mRNA levels

23 of small heterodimer partner (SHP) and protein levels of farnesoid X receptor (FXR) were

24 increased during pregnancy and lactation. Bile acid transporters Ntcp, Bsep, Mrp3 and Mrp4

25 were lower at gestation, but increased during lactation. Hepatic Oatp transporters were decreased

26 during pregnancy and lactation. Conclusion: Hepatic bile acid homeostasis is maintained during

27 normal pregnancy in rats, probably through the FXR-SHP regulation. The expression of bile acid

28 synthesis genes and liver bile acid accumulation were increased during lactation, together with

29 increased expression of bile acid efflux transporter Bsep, Mrp3 and Mrp4.

30 Keywords: Pregnant and lactating rats; Liver bile acids; Cyp7a1; FXR-SHP; Ntcp and Bsep. 


\section{Introduction}

32 Significant physiological changes occur during pregnancy and lactation to support nutritional

33 demand of the developing fetus and lactating pups (Carlin and Alfrevic, 2008; Athippozhy et al.,

34 2011). Bile acids and cholesterol metabolism are important changes during pregnancy and

35 lactation to support and to protect offspring development (Wooton-Kee, Cohen \& Vore, 2008;

36 Athippozhy et al., 2011; Abu-Hayyeh, Papacleovoulou \& Williamson, 2013a). Such

37 physiological changes would also affect hepatic drug processing genes of phase-1, phase-2

38 metabolism and transporters (Aleksunes et al., 2012; Shuster et al., 2013). The alteration of bile

39 acid homeostasis during pregnancy could unmask cholestatic disease in genetically predisposed

40 but otherwise asymptomatic individuals (Milona et al., 2010). Recent work suggests that in

41 pregnant mice farnesoid X receptor (FXR)-SHP (small heterodimer partner, NR0B2) regulation

42 could be dysfunctional in its ability to down-regulate the rate-limiting bile acid synthetic enzyme

43 Cyp7a1 and 8b1, resulting in bile acids accumulation in the liver of late pregnancy mice (Milona

44 et al., 2010; Aleksunes et al., 2012).

45 Intrahepatic cholestasis of pregnancy (ICP) is a liver disease which can occur in the third

46 trimester of pregnancy (Abu-Hayyeh, Papacleovoulou \& Williamson, 2013a). The etiology and

47 pathogenesis of ICP are still not clear, but many studies have related this disease to abnormal bile

48 acid metabolism (Abu-Hayyeh, Papacleovoulou \& Williamson, 2013a; Floreani, et al., 2013).

49 ICP with elevated bile acids in serum and liver is a major cause for premature embryo

50 development and embryonic death (Diken et al., 2013). Genetic variations or mutations of

51 farnesoid X receptor (FXR) (Van Mil et al., 2007), bile salt export pump (BSEP/ABCB11) (Dixon 
52 et al., 2009), and ATP-binding cassette, sub-family B (MDR/TAP), member 4 (ABCB4/MDR3)

53 and ABCB11 (Dixon et al., 2000; Anzivino et al., 2013) contribute to the etiology of ICP. To

54 fully understand bile acid synthesis, transport, and regulation in normal pregnancy would help us

55 to shed light on the pathology of ICP.

56 Estradiol and/or its metabolites may interfere with FXR activity during pregnancy (Milona et

57 al., 2010; Aleksunes et al., 2012), and a defect in progesterone metabolism is also implicated in 58 the etiology of ICP (Pascual et al., 2002). Estrogen signaling is associated with 59 pregnancy-induced hepatotoxicity and cholestasis in mice (Arrese et al., 2008), and reduced 60 hepatic PPAR- $\alpha$ function in the mouse also appears to be estrogen-dependent (Papacleovoulou, 61 Abu-Hayyeh \& Willamson, 2011).

62 The above scenario has been studied extensively in mice (Milona et al., 2010; Aleksunes et al., 63 2012; Shuster et al., 2013). Mice and rats are two most commonly used experimental animals, but 64 some physiological responses are different. For example, in mice, Cyp7a1 and liver bile acid pool 65 were not increased during lactation (Aleksunes et al., 2012), whereas the bile acid synthesis gene 66 Cyp7a1 and hepatic bile acids are increased 2-3 fold in lactating rats (Wooton-Kee, Cohen \& 67 Vore, 2008; Wooton-Kee et al., 2010). In mice, pregnancy and lactation are associated with 68 decreases in hepatic transporters, including bile acid transporters (Aleksunes et al., 2012), and 69 such a phenomenon should also be characterized in rats. To fully understand bile acid synthesis, 70 transport, and regulation in normal pregnancy would help us to shed light on the pathology of

71 ICP. This study was initiated to investigate bile acid metabolism and transport gene expressions 72 in pregnant and lactating rats, and the results suggest that under physiological conditions, 
73 FXR-SHP regulation might play roles in bile acid homeostasis in pregnant and lactating rats.

\section{Materials and Methods}

75 Animals. Adult Sprague Dawley (SD) rats (250 g) were purchased from the Experimental

76 Animal Center of Third Military Medical College (Chongqing, China; certificate No CXK

77 2007-0005). Rats were kept in a SPF-grade animal facilities (certificate No SYXK 2011-004) at

78 Zunyi Medical College, with regulated environment $\left(22 \pm 1^{\circ} \mathrm{C}, 50 \pm 2 \%\right.$ humidity and a 12 h: 12

79 h light: dark cycle) and free access to purified water and standard rodent chow. Rats were

80 acclimatized for 1 week, and subjected to timely mating overnight. A vaginal plug in the next

81 morning was designated as day 0 (GD 0) of gestation. Maternal livers were collected without

82 fasting on GD10, GD14 and GD19, as well as on the postnatal days (PND) 1, 7, 14 and 21. The

83 age-matched virgin rats were used as controls. The experimental design followed similar time

84 points in mice (Aleksunes et al., 2012). Livers were weighed, snap frozen in liquid nitrogen, and

85 stored at $-80^{\circ} \mathrm{C}$ until analysis. All animal procedures follow the NIH guide of Humane Use and

86 Care Animals, and approved by Institutional Animal Use and Care Committee of Zunyi Medical

87 College

\section{Bile acid determination}

89 Bile acids were extracted from the liver and measured with the "Total” Bile Acid assay (TBA)

90 kit (Nanjing Jian-Cheng Bioengineering Co., China). Briefly, livers were homogenized in

91 physiological saline (1:9, wt :vol ), followed by centrifugation at $2500 \mathrm{rpm} / \mathrm{min}$ for $10 \mathrm{~min}$. the 
92 supernatant $(30 \mu \mathrm{l})$ was taken for determination of bile acids according to manufacturer's

93 protocol.

\section{RNA Isolation and real-time RT-PCR analysis}

95 Total RNA was isolated from frozen liver sample (50-100 mg) using $1 \mathrm{ml}$ TRIzol (Takara,

96 Biotechnology, Dalian, China) and subsequently purified with Total RNA (Mini) Kit (Watson

97 Biotechnology, Shanghai, China). The quality of purified RNA was determined by

98 spectrophotometry with the 260/280 ratio $>1.8$. Purified RNA was reversed transcribed with the

99 High Capacity Reverse Transcriptase Kit (Applied Biosystems, Foster City, CA, USA). The

100 primer pairs were designed with the Primer3 software and listed in Supplementary Table 1. The

101 Power SYBR Green Master Mix (Applied Biosystems, Foster City, CA, USA) was used for

102 real-time RT-PCR analysis. The cycle time for reaching threshold (Ct) of each target gene was

103 normalized to the housekeeping genes (G3PDH and $\beta$-actin), and expressed as \% of

104 housekeeping genes.

105 Western Blot Analysis

106 Livers were homogenized in RIPA lysis buffer (Beyotime, P0013B, Shanghai, China)

107 containing freshly-prepared proteinase inhibitors. The supernatants were centrifuged at 12000

$108 \mathrm{rpm} 10 \mathrm{~min}$ at $4^{\circ} \mathrm{C}$, and protein concentrations were quantified by the BCA assay (Beyotime,

109 P0012, Shanghai, China). Aliquot proteins were denatured with protein loading buffer (Beyotime,

110 P0015, Shanghai, China), and approximately $50 \mu \mathrm{g}$ of protein/lane was separated on 10\%

111 SDS-PAGE and transferred to PVDF membranes. Membranes were blocked in 5\% non-fat milk 
112 in TBST, followed by incubation overnight at $4{ }^{\circ} \mathrm{C}$ with 1:1000 CYP7A1 (ab65586) and $\beta$-actin

113 (Ab8227) from Abcam (Cambridge, MA), or FXR (sc-13063) from Santa Cruz Biotechnology

114 (Santa Cruz, CA) in 1\% BSA. After washing with TBST three times, the membranes were

115 incubated with HRP-conjugated anti-rabbit or anti-mouse IgG (Beyotime, A0208 and A0216,

116 Shanghai, China). Protein-antibody complexes were visulized using an enhanced

117 chemiluminescent reagent (ECL-Plus) (Beyotime, P0018, Shanghai, China), and exposed to Gel

118 Imaging (Bio-Rad, ChemiDoc XRS, USA). The intensity of the band was semi-quantified with

119 Quantity One software.

120 Statistical Analysis

121 The software SPSS17.0 was used for statistical analysis. Results were described using mean \pm

122 SEM. Difference between virgin and pregnant rats was determined by two-tailed independent

123 samples test, $\mathrm{P}<0.05$ was considered statistically significant.

\section{Results}

\section{Liver bile acid levels in pregnant and lactating rat}

126 Bile acids were quantified in livers from control and pregnant rats at GD10, 14, and 19 and

127 PND 1, 7, 14, and 21. Liver bile acid levels slightly decreased in late pregnancy, especially on

128 GD 10, and 19. After birth, liver bile acid concentrations tended to increase, and there is a

129 significant increase in PND 21 (30\% over control) (Fig.1).

130 Hepatic mRNA expression of bile acid synthesis genes in pregnant and lactating rat.

131 Expression of the classic pathway bile acid synthetic enzyme genes (Cyp7a1 and 8b1) and 
132 alternative pathway (Cyp27a1 and 7b1), is shown in Fig.2. The expression of rate-limiting

133 Cyp7a1 mRNA was unchanged during pregnancy, and increased on postpartum. Cyp8b1 mRNA

134 decreased in GD10 and GD14, and increased about 2-fold in PND14. The expression of

135 alternative pathway genes Cyp27a1 and Cyp7b1 were unchanged in gestation days and increased

136 in postnatal days.

137 Hepatic expression of bile acid synthetic rate-limiting protein Cyp7A1 in pregnant and 138 lactating rat.

139 Western bolts were performed using liver homogenates from control rats, pregnant rats at GD

140 10, 14, 19 and lactating rats at PND 1, 7, 14 and 21. The expressions of CYP7A1 protein were

141 semi-quantified by band intensity. CYP7A1 protein was basically unchanged during pregnancy, a

142 result similar to Cyp7a1 mRNA expression, but increased on lactation days PND7, 14 and 21

143 (Fig. 3).

144 Hepatic mRNA expression of nuclear receptors FXR, SHP, and ESR-1, PPAR- $\alpha$ in pregnant

145 and lactating rat

146 The expression of bile acid regulation nuclear receptor genes farnesoid X receptor (FXR,

147 NR1H4) did not show significant increases during pregnancy, while FXR gradually increased on

148 postpartum. The small heterodimer partner (SHP; NR0B2) significantly increased in the late

149 gestational days, increased 3-fold on GD 19 as compared to controls. FXR plays an important

150 role in bile acid homeostasis by inducing the transcription repressor SHP (Chiang, 2009).

151 Estrogen receptor alpha (ESR-1) decreased to $64.7 \%$ and $57.7 \%$ on GD10 and GD14. In

152 postnatal days, ESR-1 increased 2.33-fold in PND1 and then decreased to 68\% of control on 
153 PND21. Proliferator-activated receptor $\alpha(\operatorname{PPAR} \alpha)$ increased 3.79-fold compared to controls

154 during lactation.

155 Hepatic expression of FXR protein in pregnant and lactating rat.

156 Western blots were performed using liver homogenates from control rats, pregnant rats at GD

157 10, 14, 19 and lactating rats at PND 1, 7, 14 and 21. The expression of FXR protein was

158 semi-quantified by band intensity. FXR protein was increased during late pregnancy (GD10 to

159 GD19) and early lactation (PND1 to PND7) (Fig. 5).

\section{Hepatic mRNA expression of bile acid transporters in pregnant and lactating rats.}

161 As illustrated in Fig 6, the expression of bile acid efflux transporter bile salt export pump

162 (Bsep/ABCB11) was decreased during pregnancy but increased during lactation. The multidrug

163 resistance protein 3 (Mrp3) and Mrp4 showed the similar pattern, with slightly increases during

164 lactation. The ATP-binding cassette sub-family G member 2 (Abcg2/BCRP) was also decreased

165 in the gestation and lactation days expect PND1.

166 Hepatic mRNA expression of uptake OATP transporters and Ntcp in pregnant and

\section{7 lactating rats.}

168 Figure 7 demonstrates that the expression of canalicular uptake transporter solute carrier 169 organic anion transporter (Oatp1/Slco1a1), solute carrier organic anion transporter 170 (Oatp2/Slco1b2), and organic anion-transporting polypeptide 4 (Oatp4/Slc21a10) were all

171 decreased in the gestation days, on PND1 Oatp1 increased 1.68-fold, then decreased in postnatal 172 days. In comparison, Oatp2 and Oatp4 decreased in the both gestation days and lactation days. 
173 The uptake transporter Na+-taurocholate co-transporting polypeptide (Ntcp) was also decreased

174 during pregnancy, increased on PND1, but decreased again thereafter during lactation.

\section{Discussion}

176 The present study demonstrates that in pregnant rats, hepatic bile acids were not elevated.

177 Consistent with hepatic bile acid concentrations, bile acid synthesis genes and/or enzymes, i.e.,

178 Cyp7a1, Cyp8b1, Cyp27a1 and Cyp7b1 were not increased during pregnancy. Increased FXR

179 protein and SHP mRNA are associated with bile acid homeostasis during pregnancy. In

180 comparison, lactating rats had increased liver bile acid, increased bile acid synthetic enzymes,

181 and increased expression of bile acid efflux transporters. In general, OATP transporters and bile

182 acid uptake transport Ntcp were down-regulated during pregnancy and lactation in rats.

183 ICP is characterized by raised serum bile acid levels and abnormal liver function tests (Geenes

184 \& Williamson, 2009; Diken, Usta \&Nassar, 2013). However, in normal pregnant women, serum

185 bile acid levels are not necessarily increased during pregnancy, regardless of gestation days

186 (Barth et al., 2005; Egan et al., 2012). In experimental animal studies, a mild increase in liver bile

187 acid levels during normal pregnancy in mice was reported in some studies (Aleksunes et al.,

188 2012), but not in others (Abu-Hayyeh, Papacleovoulou \& Williamson, 2013a). In the majority

189 cases such mild increases do not reach pathological levels and remain below the upper end of the

190 reference range for serum bile acid levels (Abu-Hayyeh, Papacleovoulou \& Williamson, 2013a).

191 Thus, it is not surprising that in the present study, liver bile acids were not elevated during

192 pregnancy in gestation days (Fig. 1). The expression of bile acid synthesis gene and proteins

193 during the gestation days (Fig. 2 and 3) is in agreement with hepatic bile acid profiles.

194 ICP has a complex etiology including genetic factors, endocrine factors, and the impact of 
pregnancy on FXR function (Abu-Hayyeh, Papacleovoulou \& Williamson, 2013a; Floreani, et,

196 al., 2013). The present study focused on FXR-SHP regulation under physiological conditions. It

197 is proposed that pregnancy in mice resembles a state of FXR inactivation (Milona et al., 2010;

198 Aleksunes et al., 2012), and attenuated FXR function during mouse pregnancy has been reported 199 (Papacleovoulou, Abu-Hayyeh \& Williamson, 2011;Aleksunes et al., 2012) and the 3ß-sulfated 200 progesterone metabolite epiallopregnanolone sulfate was found to inhibit FXR, resulting in 201 reduced FXR-mediated bile acid efflux (Abu-Hayyeh et al., 2013b). In the present study, the 202 expression of FXR mRNA in rats during pregnancy was basically unchanged. However, the FXR 203 protein and FXR-inducible negative target SHP were markedly increased at the late gestation 204 days and reached approximately 3-fold higher at GD14 and GD19, despite FXR mRNA was not 205 increased. The increases in FXR-SHP may play important role in maintaining the bile acid 206 homeostasis and preventing the liver bile acids to accumulate to protect the fetus from the bile 207 acid toxicity.

208 Lactation is a time of a five-fold increase in energy demand, as suckling young requires a 209 proportional adjustment in the ability of the lactating dam to absorb nutrients (Cripps \&

210 Williams, 1975; Vernon et al., 2002). Lactating rats have a two to three-fold increase in food

211 consumption to ensure lactating dams to absorb nutrients and to synthesize critical molecules

212 including bile acids to meet the dietary needs of the offspring and the dam (Vernon et al., 2002).

213 The size and hydrophobicity of the bile acid pool increase during lactation, implying an increased

214 absorption and disposition of lipid, sterols, nutrients, and xenobiotics (Athippozhy et al., 2011).

215 In essence, rats (Wooton-Kee, Cohen \&Vore, 2008) are different from mice (Aleksunes et al., 216 2012) in bile acid homeostasis during lactation. In the present study, hepatic bile acid pool (Fig. 
217 1), mRNA levels of bile acid synthesis gene Cyp7a1, Cyp8b1, Cyp27a1 and Cyp7b1 (Fig. 2 and

218 Fig. 3) were all increased during lactation, consistent with this scenario.

219 The mRNA levels of bile acid transporters Ntcp and Bsep followed the similar pattern. Ntcp is

220 the major bile acid transporter for conjugated bile acid (Csanaky et al., 2011) and Bsep is the

221 major bile acid efflux pump located at the bile canalicular apical domain of hepatocytes (Lam,

222 Soroka \& Boyer, 2010). Down-regulation of Ntcp and Bsep was observed in pregnant rats

223 (Arrese et al., 2003; Cao et al., 2001), however, they are increased on early postpartum, probably

224 under the influence of prolactin (Cao et al., 2001). In the present study, the changes in mRNA

225 levels of Ntcp and Bsep showed a similar pattern, i.e., lower expression during pregnancy but

226 returned to normal and even increased during lactation.

227 Sulfated progesterone metabolite (P4-S) levels are raised in normal pregnancy and elevated

228 further in ICP, which can cause a competitive inhibition of NTCP-mediated uptake of

229 taurocholate in Xenopus oocytes (Abu-Hayyeh et al., 2010), and also can cause inhibition of

230 BSEP (Vallejo et al., 2006). In the present study, mRNA levels of Ntcp and Besp (Fig. 5) were

231 lower during pregnancy, and Bsep was increased during lactation, consistent with liver bile acid

232 homeostasis profile. Mrp3 and Mrp4 are two major bile acid efflux (Cui et al., 2009; Aleksunes

233 et al., 2012), and mRNA levels of these two genes expression showed the similar pattern (Fig. 5),

234 i.e., lower during the pregnancy and higher during lactation. The pattern of these transporter

235 mRNA levels coincide with FXR-SHP regulation of bile acid homeostasis, and fortifying the

236 concept that under physiological conditions, FXR-SHP regulation of bile acid synthesis could be

237 essential for maintaining the bile acid homeostasis and could prevent the occurrence of ICP, an

238 unusual pathological condition. 
239 One of the major findings in the study is the decreased mRNA levels of Oatp transporters (Fig.

240 6), and this finding is consistent with that observed in mice (Aleksunes et al., 2012; Shuster et al.,

241 2013). Oatps are important not only for bile acid transport (Zhang et al., 2012), but also

242 important for drugs and xenobiotic transport (Lu et al., 2008). In pregnant rats, the expression of

243 Oatp2, but not Oatp1, was reported to decrease (Cao et al., 2001; 2002). The generalized

244 down-regulation of Oatp transporters could be an adaptive mechanism for dam to protect

245 developing fetus and nursing pups from toxicants. Abcg2 is involved in epithelial

246 transport/barrier functions, including bile acid transport (Blazquezet al., 2012). Abcg2 is

247 proposed to play a key role in bile acid transport in placenta, as Bsep does in liver (Blazquez et

248 al., 2012). In the present study, mRNA levels of Abcg 2 was depressed during pregnancy and

249 lactation except for a transient increase at PND1. The pattern of Abcg2 expression is similar to

250 Oatps, and can also be envisioned as an adaptive mechanism during pregnancy and lactation.

251 In summary, the present study suggests that in pregnant rats, FXR-SHP could regulate bile acid

252 synthesis enzyme genes to prevent the accumulation of bile acids in the liver, together with

253 down-regulation of bile acid transporters Ntcp and Bsep. Pregnancy and lactation is associated

254 with a general down-regulation of Oatp and Abcg2 in rats. These data would add to our

255 understanding of FXR-SHP regulation of bile acid homeostasis under physiological conditions. 
257

258

259

260

261

262

263

264

265

266

267

268

269

270

271

272

273

274

275

276

277

278

279

280

281

282

283

284

285

286

287

288

289

290

291

292

293

294

295

296

297

Abu-Hayyeh S, Martinez-Becerra P, Sheikh Abdul Kadir SH, Selden C, Romero MR, Rees

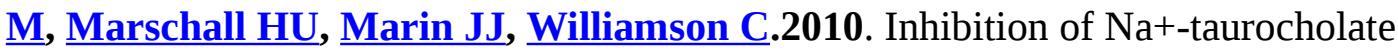
Co-transporting polypeptide-mediated bile acid transport by cholestatic sulfated progesterone metabolites. The Journal of Biological Chemistry 285:16504-16512.

Abu-Hayyeh S, Papacleovoulou G, Williamson C.2013a.Nuclear receptors, bile acids and cholesterol homeostasis series - Bile acids and pregnancy. Molecular and Cellular Endocrinology 368:120-128.

Abu-Hayyeh S, Papacleovoulou G, Lövgren-Sandblom A, Tahir M, Oduwole O, Jamaludin NA, Ravat S, Nikolova V, Chambers J, Selden C, Rees M, Marschall HU, Parker MG, Williamson C. 2013b.Intrahepatic cholestasis of pregnancy levels of sulfated progesterone metabolites inhibit farnesoid $\mathrm{X}$ receptor resulting in a cholestatic phenotype. Hepatology 57:716-726.

Aleksunes LM, Yeager RL, Wen X, Cui JY, Klaassen CD. 2012.Repression of hepatobiliary transporters and differential regulation of classic and alternative bile acid pathways in mice during pregnancy. Toxicological Sciences 130:257-268.

Anzivino C, Odoardi MR, Meschiari E, Baldelli E, Facchinetti F, Neri I, Ruggiero G, Zampino R, Bertolotti M, Loria P, Carulli L. 2013. ABCB4 and ABCB11 mutations in intrahepatic cholestasis of pregnancy in an Italian population. Digestive and Liver Disease 45:226-232.

Arrese M, Trauner M, Ananthanarayanan M, Pizarro M, Solís N, Accatino L, Soroka C, Boyer JL, Karpen SJ, Miquel JF, Suchy FJ. 2003. Down-regulation of the $\mathrm{Na}+/$ taurocholate cotransporting polypeptide during pregnancy in the rat. Journal of Hepatology 38:148-155.

Arrese M, Macias RI, Briz O, Perez MJ, Marin JJ. 2008. Molecular pathogenesis of intrahepatic cholestasis of pregnancy. Expert Reviews in Molecular Medicine 28:10:e9.

Athippozhy A, Huang L, Wooton-Kee CR, Zhao T, Jungsuwadee P, Stromberg AJ, Vore M. 2011. Differential gene expression in liver and small intestine from lactating rats compared to age-matched virgin controls detects increased mRNA of cholesterol biosynthetic genes. BMC Genomics 3:12:95.

Barth A, Rost M, Kindt A, Peiker G. 2005.Serum bile acid profile in women during pregnancy and childbed. Experimental and Clinical Endocrinology \& Diabetes 113:372-375.

Blazquez AG, Briz O, Romero MR, Rosales R, Monte MJ, Vaquero J, Macias RI, Cassio D, Marin JJ. 2012. Characterization of the role of ABCG2 as a bile acid transporter in liver and placenta. Molecular Pharmacology 81:273-283.

Cao J, Huang L, Liu Y, Hoffman T, Stieger B, Meier PJ, Vore M. 2001. Differential regulation of hepatic bile salt and organic anion transporters in pregnant and postpartum rats and the role of prolactin. Hepatology 33:140-147.

Cao J, Stieger B, Meier PJ, Vore M. 2002. Expression of rat hepatic multidrug resistance-associated proteins and organic anion transporters in pregnancy. American Journal of Physiology Gastrointestinal and Liver Physiology 283:G757-G766.

Carlin A, Alfirevic Z.2008.Physiological changes of pregnancy and monitoring. Best Practice \& 

research. Clinical Obstetrics \&Gynaecology 22:801-823.

Chiang JY. 2009. Bile acids: regulation of synthesis. Journal of Lipid Research 50:1955-1966.

Cripps AW, Williams VJ. 1975. The effect of pregnancy and lactation on food intake, gastrointestinal anatomy and the absorptive capacity of the small intestine in the albino rat. The British Journal of Nutrition 33:17-32.

Cui YJ, Aleksunes LM, Tanaka Y, Goedken MJ, Klaassen CD. 2009.Compensatory induction of liver efflux transporters in response to ANIT-induced liver injury is impaired in FXR-null mice. Toxicological Sciences 110: 47-60.

Csanaky IL, Lu H, Zhang Y, Ogura K, Choudhuri S, Klaassen CD. 2011.Organic anion-transporting polypeptide $1 \mathrm{~b} 2$ (Oatp1b2) is important for the hepatic uptake of unconjugated bile acids: Studies in Oatp1b2-null mice. Hepatology 53:272-281.

Diken Z,Usta IM, Nassar AH. 2013. A Clinical Approach to Intrahepatic Cholestasis of Pregnancy. American Journal of Perinatology 2013 Jan 28 [Epub ahead of print].

Dixon PH, Weerasekera N, Linton KJ, Donaldson O, Chambers J, Egginton E, Weaver J, Nelson-Piercy C, de Swiet M, Warnes G, Elias E, Higgins CF, Johnston DG, McCarthy MI, Williamson C. 2000. Heterozygous MDR3 missense mutation associated with intrahepatic cholestasis of pregnancy: evidence for a defect in protein trafficking. Human Molecular Genetics 9:1209-1217.

Dixon PH, van Mil SW, Chambers J, Strautnieks S, Thompson RJ, Lammert F, Kubitz R, Keitel V, Glantz A, Mattsson LA, Marschall HU, Molokhia M, Moore GE, Linton KJ, Williamson C. 2009. Contribution of variant alleles of ABCB11 to susceptibility to intrahepatic cholestasis of pregnancy. Gut 58:537-544.

Egan N, Bartels A, Khashan AS, Broadhurst DI, Joyce C, O'Mullane J, O'Donoghue K. 2012. Reference standard for serum bile acids in pregnancy. BJOG 119:493-8.

Floreani A, Caroli D, Lazzari R, Memmo A, Vidali E, Colavito D, D'Arrigo A, Leon A, Romero R, Gervasi M. 2013. Intrahepatic Cholestasis Of Pregnancy: New Insights Into Its Pathogenesis. The Journal of Maternal-Fetal\& Neonatal Medicine. 2013 Mar 12 [Epub ahead of print]

Geenes V, Williamson C. 2009. Intrahepatic cholestasis of pregnancy. World Journal of Gastroenterology15:2049-2066.

Lam P, Soroka CJ, Boyer JL. 2010. The bile salt export pump: clinical and experimental aspects of genetic and acquired cholestatic liver disease. Seminars in Liver Diseases 30:125-133.

Lu H, Choudhuri S, Ogura K, Csanaky IL, Lei X, Cheng X, Song PZ, Klaassen CD. 2008. Characterization of organic anion transporting polypeptide 1b2-null mice: essential role in hepatic uptake/toxicity of phalloidin and microcystin-LR. Toxicological Sciences 103:35-45.

Milona A, Owen BM, Cobbold JF, Willemsen EC, Cox IJ, Boudjelal M, Cairns W, Schoonjans K, Taylor-Robinson SD, Klomp LW, Parker MG, White R, van Mil SW, Williamson C. 2010.Raised hepatic bile acid concentrations during pregnancy in mice are associated with reduced farnesoid X receptor function. Hepatology 52:1341-1349.

Papacleovoulou G, Abu-Hayyeh S, Williamson C. 2011. Nuclear receptor-driven alterations in bile acid and lipid metabolic pathways during gestation. Biochimica et Biophysica Acta 1812:879-887. 
341 Pascual MJ, Serrano MA, El-Mir MY, Macias RI, Jiménez F, Marin JJ. 2002. Relationship 342 between asymptomatic hypercholanaemia of pregnancy and progesterone metabolism. $\underline{\text { Clinical }}$ 343 Science 102:587-593.

344 Shuster DL, Bammler TK, Beyer RP, Macdonald JW, Tsai JM, Farin FM, Hebert MF, 345 Thummel KE, Mao Q. 2013. Gestational age-dependent changes in gene expression of 346 metabolic enzymes and transporters in pregnant mice. Drug Metabolism and Disposition 347 41:332-342.

348 Vallejo M, Briz O, Serrano MA, Monte MJ, Marin JJ. 2006. Potential role of trans-inhibition 349 of the bile salt export pump by progesterone metabolites in the etiopathogenesis of 350 intrahepatic cholestasis of pregnancy. Journal of Hepatology 44:1150-1157.

351 Van Mil SW, Milona A, Dixon PH, Mullenbach R, Geenes VL, Chambers J, Shevchuk V, 352 Moore GE, Lammert F, Glantz AG, Mattsson LA, Whittaker J, Parker MG, White R, 353 Williamson C. 2007. Functional variants of the central bile acid sensor FXR identified in 354 intrahepatic cholestasis of pregnancy. Gastroenterology 133::507-516.

355 Vernon RG, Denis RG, Sorensen A, Williams G. 2002.Leptin and the adaptations of lactation 356 in rodents and ruminants. Hormone Metabolic Research 34:678-685.

357 Wooton-Kee CR, Cohen DE, Vore M.2008. Increased cholesterol 7alpha-hydroxylase 358 expression and size of the bile acid pool in the lactating rat. AmericanJournal of Physiology $359 \quad$ Gastrointestinal and Liver Physiology 294:G1009-G1016.

360 Wooton-Kee CR, Coy DJ, Athippozhy AT, Zhao T, Jones BR, Vore M. 2010. Mechanisms for 361 increased expression of cholesterol 7alpha-hydroxylase (Cyp7a1) in lactating rats. Hepatology 362 51: 277-285.

363 Zhang Y, Csanaky IL, Cheng X, Lehman-McKeeman LD, Klaassen CD.2012. Organic anion 364 transporting polypeptide 1a1 null mice are sensitive to cholestatic liver injury. Toxicological 365 Sciences 127:451-462. 
367 Figure 1. Liver bile acid levels in pregnant and lactating rat

368 Bile acids were quantified in Livers from control and pregnant rat on GD10, 14, and 19 and PND

369 1, 7, 14, and 21. Dark gray bars represent pregnant rat, and black bars represent lactating rat. Data 370 are presented as mean \pm SEM (n=3-6). Asterisks $\square * \square$ represent statistically significant difference ( $371 \mathrm{p}<0.05$ ) compared with control.

372 Figure 2. Hepatic mRNA expression of bile acid synthetic pathway genes in pregnant and 373 lactating rat.

374 The expression of bile acid synthetic classic pathway genes Cyp7a1, Cyp8b1 and alternative 375 pathway genes Cyp27a1 and Cyp7b1 was quantified from control and GD10,14 and 19 and PND

376 1,7,14 and 21, Data were normalized to controls (set to 100\%) and presented as mean \pm SEM 377 ( $n=3-6)$. Dark gray bars represent pregnant rat, and black bars represent lactating rat. Asterisks $378 \square * \square$ represent statistically significant difference $(\mathrm{p}<0.05)$ compared with control.

379 Figure 3. Hepatic expression of bile acid synthesis rate-limiting protein CYP7A1 in 380 pregnant and lactating rat.

381 Western bolts were performed using liver homogenates from control, pregnant rats in GD 10, 14, 38219 and PND 1, 7, 14 and 21. The expression of CYP7A1 was semi-quantified by band intensity. 383 Values are mean \pm SEM (n=5). Dark gray bars represent pregnant rat, and black bars represent 384 lactating rat. Significantly difference was confirmed by two-tailed independent Samples test 385 method $(\mathrm{P}<0.05)$ 
386 Figure 4. Hepatic mRNA expression of Nuclear Receptors SHP, FXR and ESR-1 and

387 PPAR- $\alpha$ in pregnant and lactating rat

388 The expression of bile acid regulation Nuclear Receptors genes SHP, FXR and Esr-1,PPAR- $\alpha$

389 were quantified using total hepatic RNA from control and pregnant mice at gestational days 10,

39014,19 and postnatal days 1, 7, 14, 21. Data were normalized to controls and presented as mean \pm

391 SEM (n=3-6). Dark gray bars represent pregnant rat, and black bars represent lactating rat.

392 Asterisks $(*)$ represent statistically significant differences $(\mathrm{p}<0.05)$ compared to control.

393 Figure 5. Hepatic expression of nuclear receptor FXR in pregnant and lactating rat.

394 Western bolts were performed using liver homogenates from control, pregnant rats in GD 10, 14,

39519 and PND 1, 7, 14 and 21. The expression of FXR was semi-quantified by band intensity.

396 Values are mean \pm SEM (n=3). Dark gray bars represent pregnant rat, and black bars represent

397 lactating rat. Significantly difference was confirmed by two-tailed independent Samples test 398 method $(\mathrm{P}<0.05)$

399 Figure 6. Hepatic mRNA expression of bile acid transporter in pregnant and lactating rats.

400 The expression of bile acid efflux transporter Bsep, Mrp3, Mrp4 and Abcg2 was quantified using 401 total hepatic RNA from pregnant rats on GD 10, 14 and 19, and postpartum rats on PND 1, 7, 14 402 and 21. Data were normalized to controls and presented as mean \pm SEM (n=3-6). Dark gray bars 403 represent pregnant rat, and black bars represent lactating rat. Asterisks (*) represent statistically 404 significant differences $(\mathrm{p}<0.05)$ compared control.

405 Figure 7. Hepatic mRNA expression of uptake transporter Oatps and Ntcp in pregnant and 
406 lactating rats.

407 The expression of hepatic canalicular uptake transporter solute carrier organic anion transporters 408 (Oatps) and $\mathrm{Na}$--taurocholate co-transporting polypeptide (Ntcp) transporters was quantified 409 using total hepatic RNA from pregnant rats on GD10, 14 and 19, and postpartum rats on PND 1, 410 7, 14 and 21. Data were normalized to controls and presented as mean \pm SEM (n=3-6). Dark gray 411 bars represent pregnant rat, and black bars represent lactating rat. Asterisks (*) represent 412 statistically significant differences $(\mathrm{p}<0.05)$ compared control. 


\section{Supplementary Table 1.}

\begin{tabular}{llll}
\hline Gene & Access & Forward & Reverse \\
\hline & & & \\
G3PDH & NM_017008.4 & AACTTTGGCATTGTGGAAGG & GGATGCAGGGATGATGTTCT \\
B-actin & NM_031144 & AGCCATGTACGTAGCCATCC & ACCCTCATAGATGGGCACAG \\
Cyp7a1 & NM_012942 & GAGAACGGGTTGATTCCGTA & AAAAACGTGACCATGCTTC \\
Cyp8b1 & NM_031241 & CACGTAGCCAGTACCAAGCA & GGTCCTAGCATCACCAAGGA \\
Cyp27a1 & NM_178847 & TCTGGCTACCTGCACTTCCT & GTCTACCCCAGCCAAGATCA \\
Cyp7b1 & NM_019183 & TCATCCGTGAAGTGCAAGAG & GGAGCATCGAAGACTTCTGG \\
FXR & NM_021745 & CGAGATGCCTGTGACAAAGA & GCAGACCACACACAGCTCAT \\
SHP & NM_053908 & TTATGTGTGAGGGTGGACGA & CCCGTCTTCTTGAAGTGCTC \\
Esr-1 & NM_012689 & TCCGGCACATGAGTAACAAA & TGAAGACGATGAGCATCCAG \\
PPAR- $\alpha$ & NM_013196 & GAGACCCTCGGGGATCTTAG & TGTGTCCTGAGCTTGACCAG \\
Ntcp & NM_017047 & CACAACGTATCAGCCCCTTT & ATGCTAAGCGCCTTGTCTGT \\
Bsep & NM_031760 & CCACCAGAACATGACAAACG & CCCAGTGATGACCCATAACC \\
MRP3 & NM_080581 & CCAGACCTCACACCCTGTTT & CGTCTTGAGCCTGGATAAGC \\
MRP4 & NM_133411 & TGAAGCAACTGCAAATGTGG & AGTGCACTGGGCAAACTTCT \\
Oatp1 & NM_017111 & GGATGTAGCTGAGGCAGAGG & CAGCTCCCAGTGGCATTTAT \\
Oatp2 & NM_0131906 & CCTAGGCATAGGCATTTGGA & TCAACCAAAGCACAAAGCAG \\
Oatp4 & NM_031650 & AACATGCTTCGTGGGATAGG & CATGGAAGTGTGCCCTTCTT \\
Abcg2 & NM_181381 & GAAAGACCCACGGGGATTAT & CCCATCACAACGTCATCTTG \\
\hline
\end{tabular}




\section{Figure 1}

Fig.1

Liver bile acid concentrations 


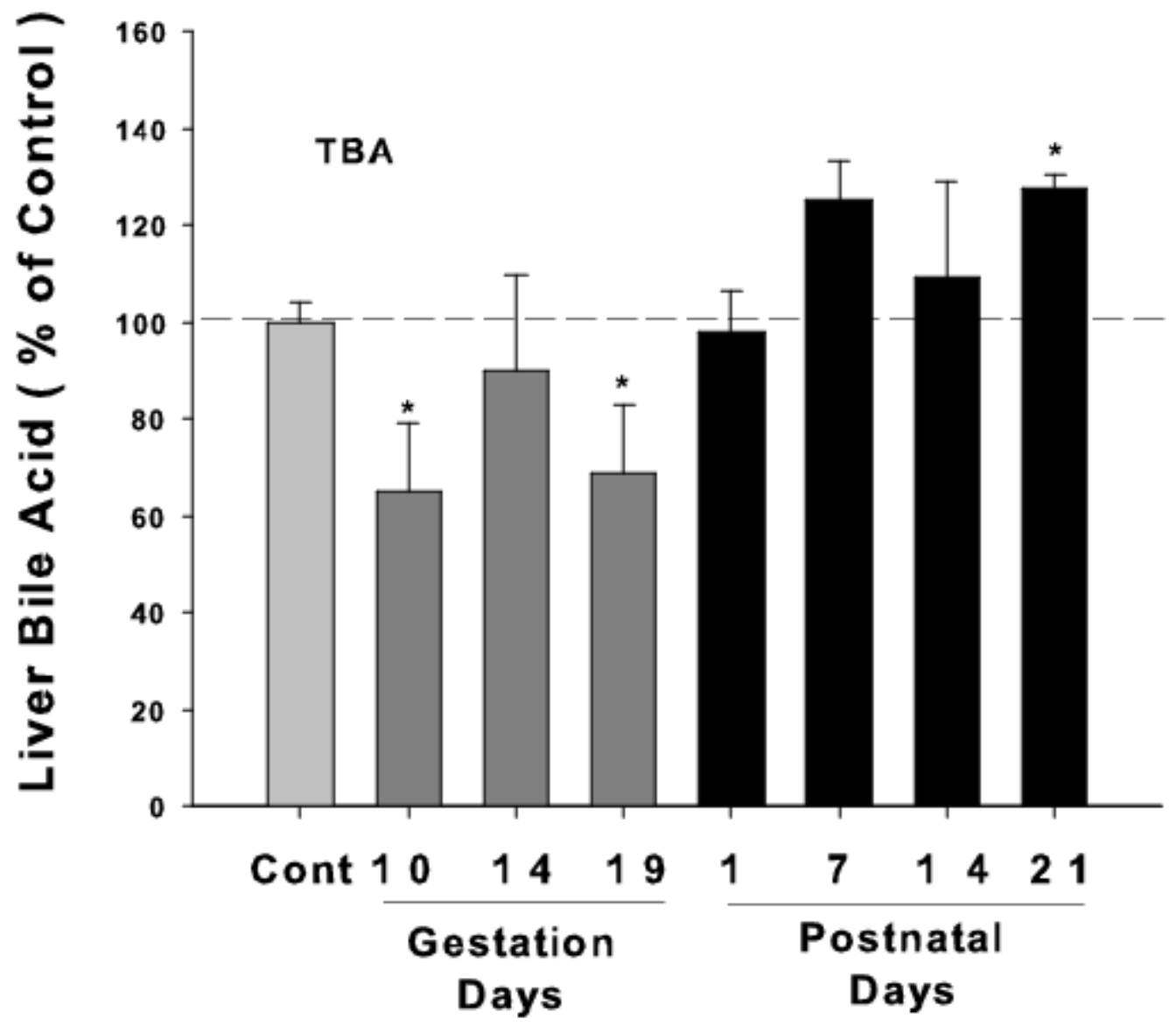

Fig.1 


\section{Figure 2}

Fig. 2

Bile acid synthesis gene expression 

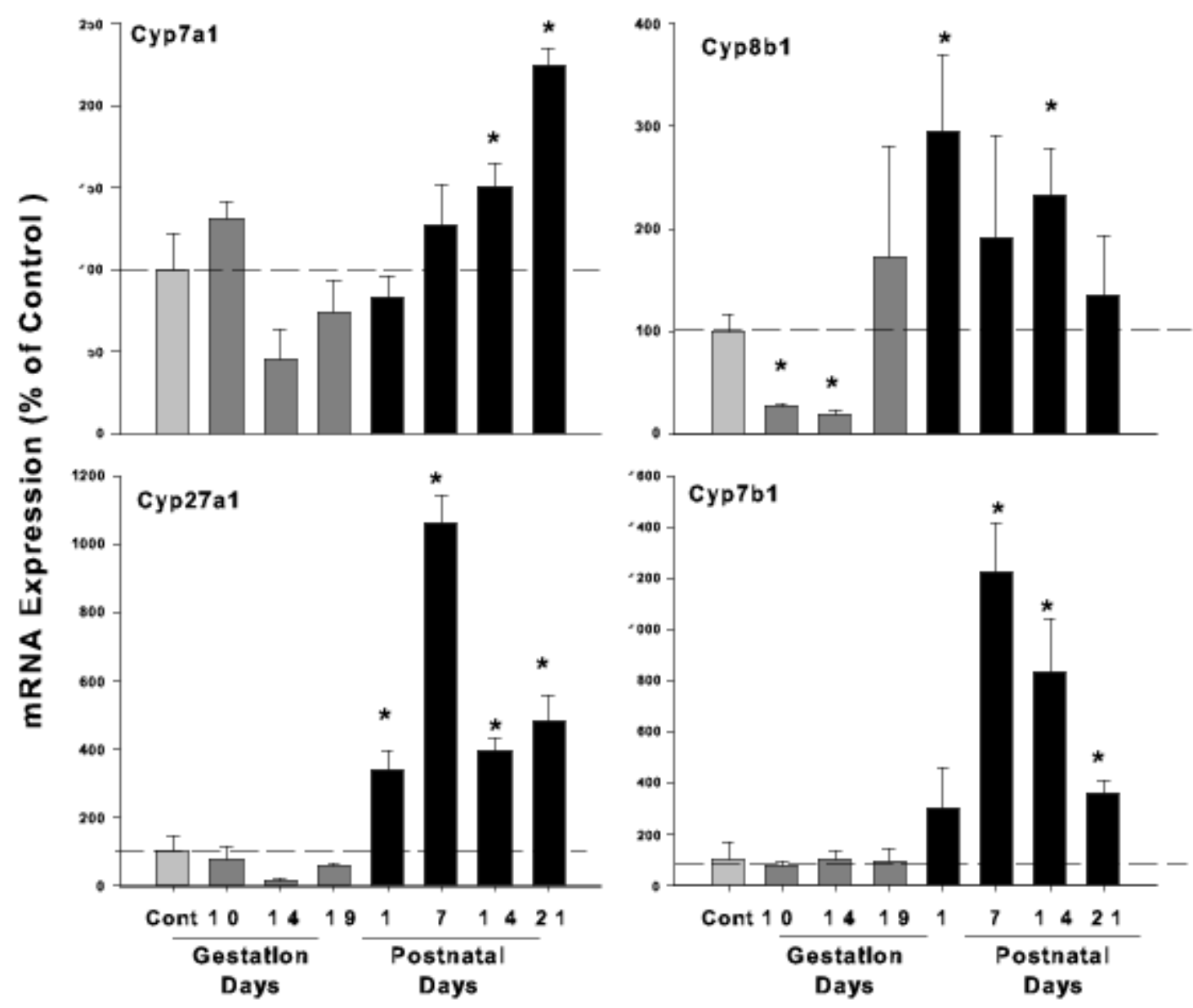

Fig.2 


\section{Figure 3}

Hepatic expression of bile acid synthesis rate-limiting protein CYP7A1 in pregnant and lactating rat.

Western bolts were performed using liver homogenates from control, pregnant rats in GD 10, 14, 19 and PND 1, 7, 14 and 21. The expression of CYP7A1 was semi-quantified by band intensity.Values are mean \pm SEM. Dark gray bars represent pregnant rat, and black bars represent lactating rat. Significant difference was confirmed by two-tailed independent Samples test method $(\mathrm{P}<0.05)$. 

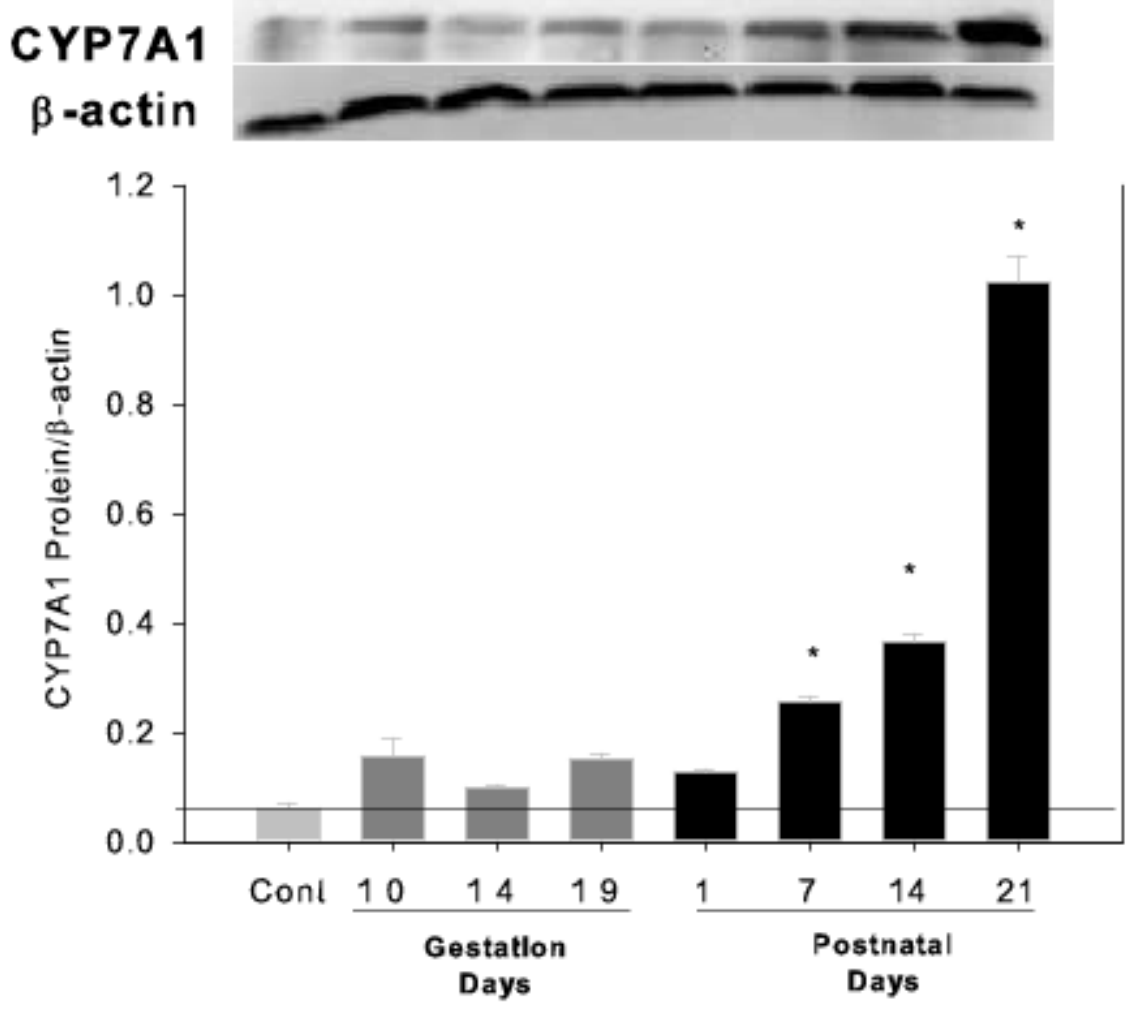


\section{Figure 4}

Fig. 4

Expression of nuclear receptors 

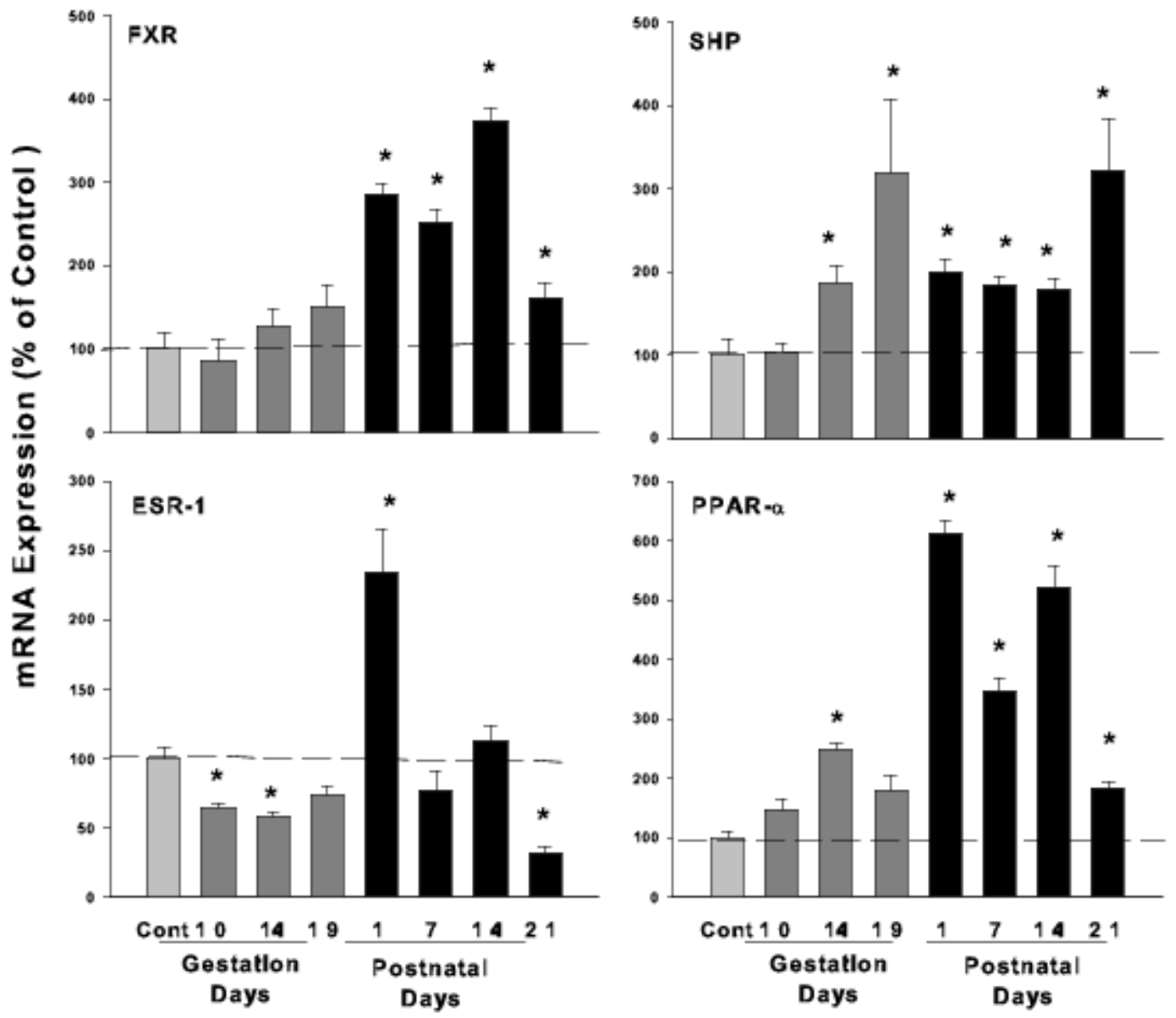

Fig.4 


\section{Figure 5}

Fig. 5

FXR protein expression

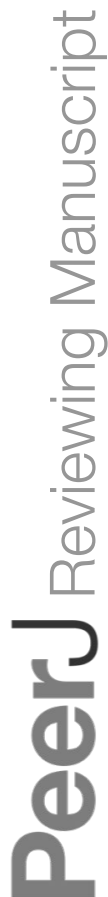

PeerJ reviewing PDF | (v2013:06:607:2:0:NEW 3 Aug 2013) 

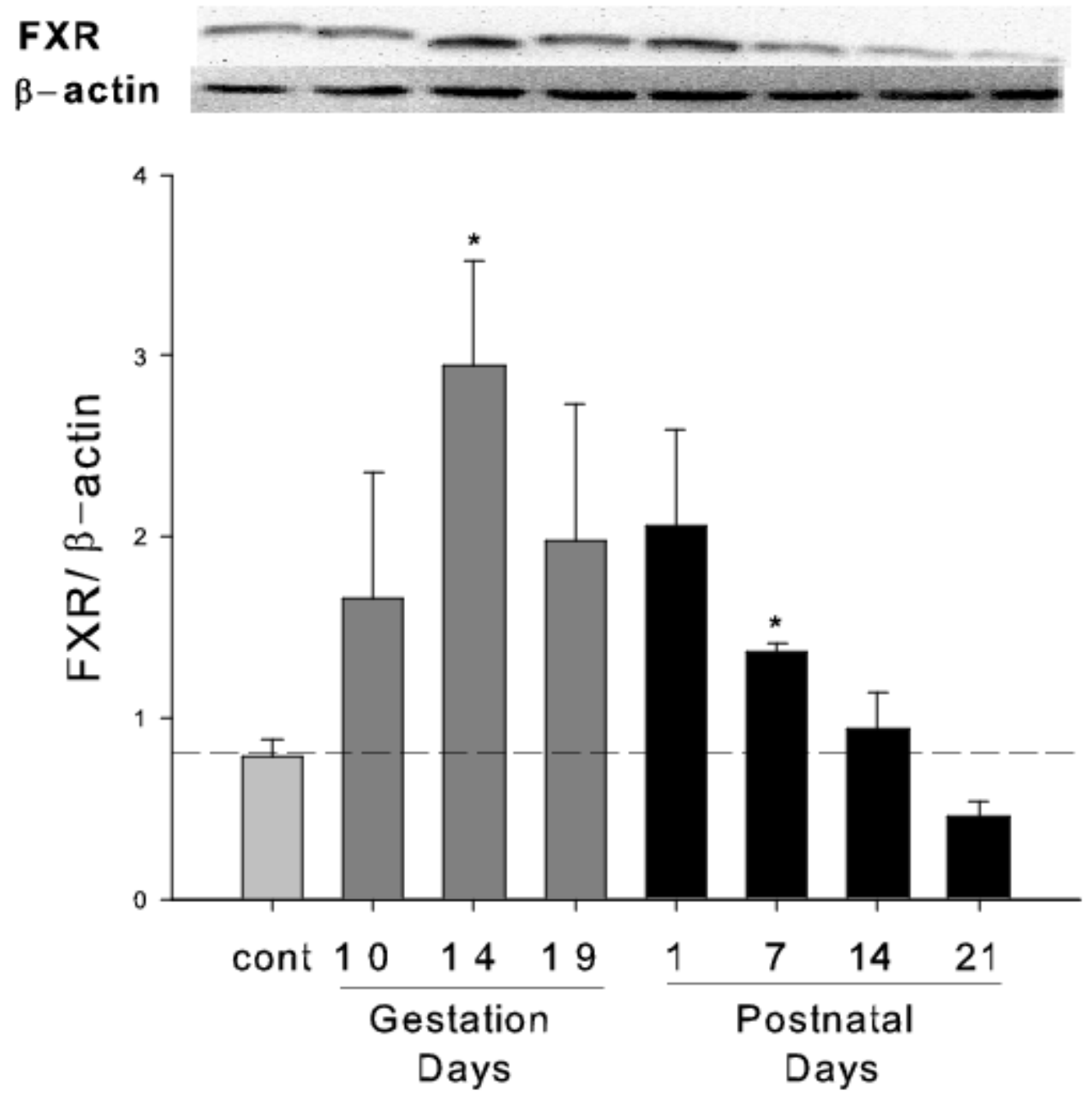

Fig.5 


\section{Figure 6}

Fig. 6

Efflux transporters

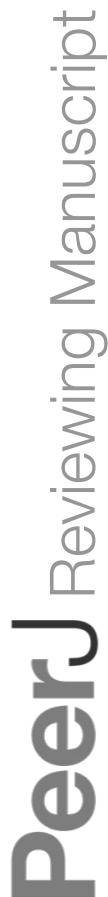

PeerJ reviewing PDF | (v2013:06:607:2:0:NEW 3 Aug 2013) 

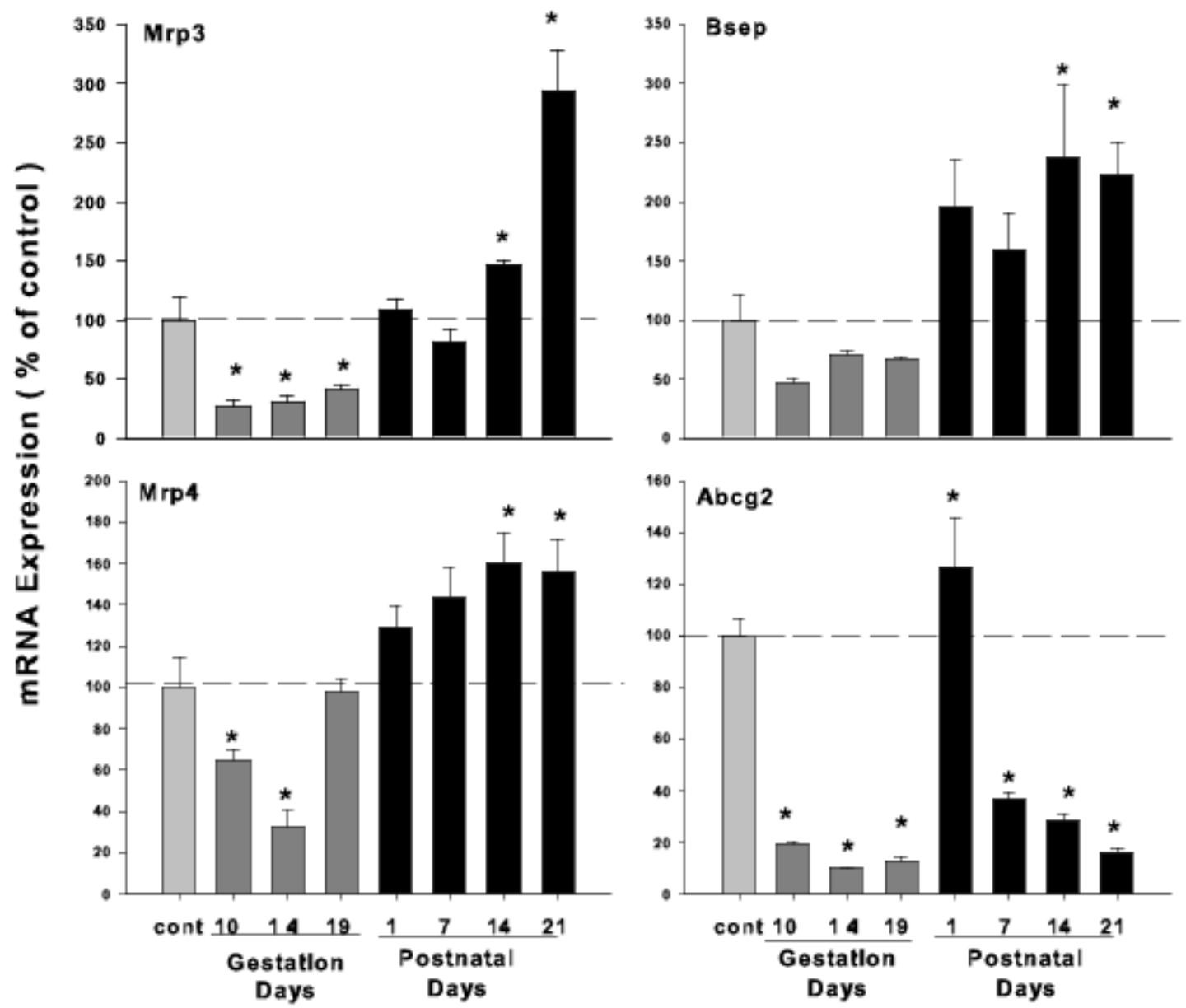

Fig. 6 


\section{Figure 7}

Fig. 7

Uptake transporter expression 

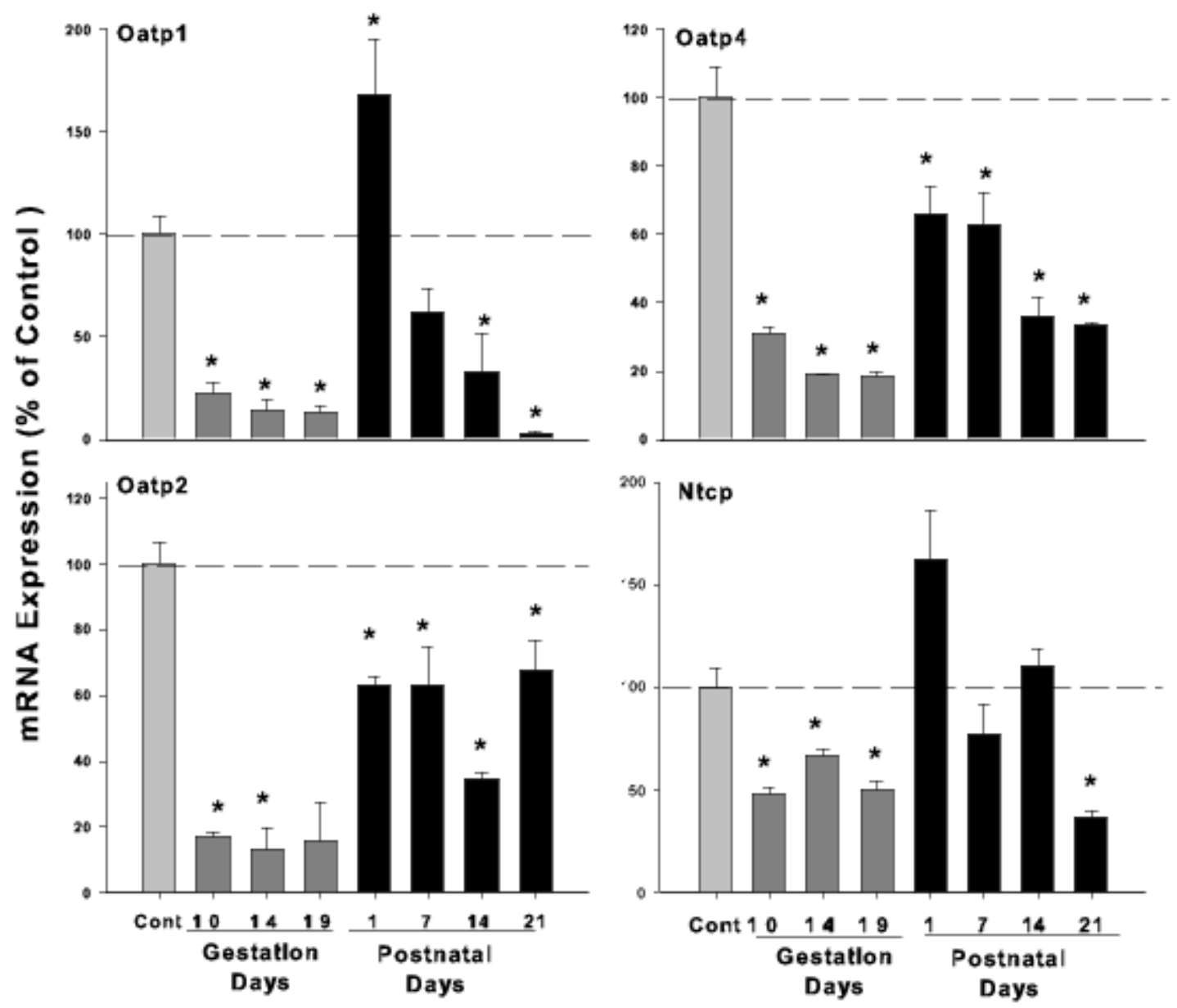

Fig.7 